\title{
MicroRNA-3907 promotes the proliferation and migration of sebaceous gland carcinoma of the eyelid by targeting thrombospondin 1
}

\author{
CHUANLI ZHANG*, LIMIN ZHU* ${ }^{*}$ XUN LIU, MEIXIA JIANG, QIN TANG, \\ FEI XU, TINGTING LIN, LIJIE DONG and YANJIN HE
}

\begin{abstract}
Tianjin Key Laboratory of Retinal Functions and Diseases, Tianjin Branch of National Clinical Research Center for Ocular Disease, Eye Institute and School of Optometry, Tianjin Medical University Eye Hospital, Tianjin 300384, P.R. China
\end{abstract}

Received June 8, 2021; Accepted September 16, 2021

DOI: 10.3892/ol.2021.13094

\begin{abstract}
MicroRNAs (miRNAs/miRs) play an important role in various types of carcinoma, including sebaceous gland carcinoma (SGC) of the eyelid. miR-3907 was found to be highly expressed in lung cancer; however, to the best of our knowledge, the biological role of miR-3907 in SGC has not previously been evaluated. The aim of the present study was to determine the role and mechanism of miR-3907 in the occurrence and development of SGC. miR-3907 was screened and identified as a novel upregulated miRNA in SGC tissues and cells, as determined using miRNA microarrays and reverse transcription-quantitative (RT-q) PCR analyses. Compared with the control group, cellular proliferation and migration were enhanced in the miR-3907 mimics group, and decreased in the miR-3907 inhibitor group. Moreover, miR-3907 negatively regulated thrombospondin 1 (THBS1) expression, as shown by bioinformatics prediction, RT-qPCR, western blotting and dual-luciferase reporter assays. In addition, compared with the control group, the small interfering (si) siRNA-THBS1 group exhibited enhanced proliferation and migration abilities, which were decreased in the THBS1 overexpression group. Furthermore, THBS1 overexpression was found to attenuate the stimulative effect of miR-3907 mimics, and THBS1-knockdown reversed the inhibitory effect of the miR-3907 inhibitor in SGC cells. Collectively, the results of the present study indicated that miR-3907 promoted the proliferation and migration of SGC by downregulating THBS1,
\end{abstract}

Correspondence to: Professor Tingting Lin, Tianjin Key Laboratory of Retinal Functions and Diseases, Tianjin Branch of National Clinical Research Center for Ocular Disease, Eye Institute and School of Optometry, Tianjin Medical University Eye Hospital, 251 Fu Kang Road, Tianjin 300384, P.R. China

E-mail: 1tt6123@126.com

*Contributed equally

Key words: sebaceous gland carcinoma, microRNA-3907, thrombospondin 1 , proliferation, migration and that this axis may be a potential target for the prognostic assessment and treatment of SGC.

\section{Introduction}

Sebaceous gland carcinoma (SGC) of the eyelid is a type of malignant epithelial tumor originating from sebaceous glands of the eyelid. SGC is the second most common malignant eyelid tumor in a number of Asian countries and ranks either third or fourth in various European countries $(1,2)$. TNM staging, larger tumor diameter, recurrence, metastasis and perivascular invasion are clinical factors for poor prognosis (3-5), and patients with SGC are prone to recurrence and metastasis after surgery, which also results in poor prognosis (6). The mortality rate is high after recurrence and metastasis, and studies have reported that SGC of eyelids ranges from 5.9 to $11.3 \%$, which may be associated with patient ethnicity, the time of follow-up and the number of samples assessed $(7,8)$. Therefore, early diagnosis and treatment are key to improving patient prognosis.

As single-stranded, non-coding small RNAs, microRNA (miRNAs/miRs) can act as cancer-promoting or tumor-suppressor genes in a variety of cancer types. In addition, studies have reported that abnormally expressed miRNAs in different carcinoma tissues can be used as biomarkers of tumor prognosis and diagnosis, including miR-200c and miR-141 in SGC $(9,10)$. miR-3907 is a novel miRNA that has been shown to be involved in lung cancer and kidney disease $(11,12)$. However, to the best of our knowledge, the role of miR-3907 in SGC of the eyelid remains unknown.

The platelet thrombin protein thrombospondin 1 (THBS1), a glycoprotein that mediates cell-to-cell and cell-to-matrix adhesion, plays an important role in a variety of diseases, including malignant tumors (13). Downregulation of THBS1 mRNA expression in laryngeal squamous cell carcinoma has been shown to be correlated with TNM stage and lymph node metastasis (14). Moreover, pigment epithelium-derived factor-induced exosomal THBS1 plays an important role in inhibiting metastasis and invasion in lung cancer (15).

To the best of our knowledge, the biological roles and expression levels of miR-3907 and THBS1 in SGC have not 
been previously investigated. Using an miRNA microarray, miR-3907 was found to be highly expressed in SGC of the eyelid. Therefore, the present study aimed to investigate the effects and potential regulatory mechanisms of miR-3907 (as a target of THBS1) on the proliferation and migration of SGC cells, with a view to providing a potential target for the early diagnosis and treatment of SGC.

\section{Materials and methods}

Tissue samples. The inclusion criteria for carcinoma tissues were that patients had undergone postoperative pathological diagnosis of eyelid SGC, whereas patients with paracarcinoma tissues had undergone an enlarged surgical resection of eyelid SGC, including the paracarcinoma normal sebaceous gland. Patients who received preoperative chemotherapy or radiotherapy were excluded from the study. A total of 18 formalin-fixed paraffin-embedded (FFPE) eyelid SGC tissues and six para-carcinoma FFPE samples were provided by the Tianjin Medical University Eye Hospital (Tianjin, China) between July 2011 and January 2019. The mean patient age was 64.4 years (range, 38-80 years), with 13 women and 5 men enrolling. In total, 10 patients were T1-T2 stage, and 8 possessed T3-T4 stage SGC. Moreover, 6 patients were histologically graded $1-2$, and 12 were graded $3-4$. Postoperative recurrence occurred in 6 patients and no recurrence occurred in 12 patients. With regards to the para-carcinoma tissues, the mean age was 63.8 years, with a range of 38-80 years. There were 4 women and 2 men in the para-carcinoma group. Written informed consent was obtained from the patients with SGC prior to tissue collection, and the study was approved by the Ethics Committee of Tianjin Medical University Eye Hospital [approval no. 2020KY(L)-20].

miRNA microarrays. Differentially expressed miRNAs in three SGC and three paired para-carcinoma control FFPE samples were detected using the Agilent Human miRNA Microarray (Agilent Technologies, Inc.). Differentially expressed miRNAs in four SGC FFPE samples containing a P53 mutation, and in four SGC FFPE samples without a P53 mutation, were also detected using the Agilent Human miRNA Microarray (Release 21.0, 8x60K; design ID, 070156) and the thresholds were set as fold change $\geq 2$ and $\mathrm{P}<0.05$.

Cell culture. Tissue block primary cell culture was performed as previously described (16). Briefly, primary cells were cultured using the tissue block culture method, in which SGC and SG samples were derived from the intraoperative tissues of patients with SGC. Following tissue removal, the specimens were immediately transferred to the laboratory for primary cell culture. The samples were rinsed 2-3 times with culture medium, cut into $1 \mathrm{~mm}^{3}$ pieces, and then slowly transferred into a T25 $\mathrm{cm}^{2}$ cell culture flask. The cells were maintained in RPMI-1640 medium (Gibco; Thermo Fisher Scientific, Inc.) supplemented with $10 \%$ FBS (Shanghai ExCell Biology, Inc.) and $1 \%$ penicillin and streptomycin (HyClone; Cytiva) at $37^{\circ} \mathrm{C}\left(5 \% \mathrm{CO}_{2}\right)$, and the medium was exchanged every 2 days. The cells were passaged at $80 \%$ confluence, and primary cells from passages $4-10$ were selected for experimental use.
$R N A$ extraction and reverse transcription-quantitative $(R T-q)$ $P C R$. All procedures were performed in strict accordance with the manufacturer's instructions. mRNA and miRNA from SGC primary cells were extracted using an EZ-press RNA Purification kit (EZBioscience). miRNA was reverse transcribed into first-strand cDNA using the miRcute Plus miRNA First-Strand cDNA kit (Tiangen Biotech Co., Ltd.) at $42^{\circ} \mathrm{C}$ for $60 \mathrm{~min}$ and $95^{\circ} \mathrm{C}$ for $3 \mathrm{~min}$. miRNA SYBR Green analysis was performed using the miRcute Plus miRNA qPCR kit (Tiangen Biotech Co., Ltd.) under the following thermocycling conditions: Initial denaturation at $95^{\circ} \mathrm{C}$ for $15 \mathrm{~min}$; followed by 40 cycles of $95^{\circ} \mathrm{C}$ for $20 \mathrm{sec}$ and $60^{\circ} \mathrm{C}$ for $30 \mathrm{sec}$. The first-strand cDNA from mRNA was obtained using the FastKing RT kit (Tiangen Biotech Co., Ltd.) per the following conditions: $42^{\circ} \mathrm{C}$ for $3 \mathrm{~min}, 42^{\circ} \mathrm{C}$ for $15 \mathrm{~min}$ and $95^{\circ} \mathrm{C}$ for $3 \mathrm{~min}$. mRNA SYBR-Green quantification analysis was performed using the SuperReal PreMix Plus kit (Tiangen Biotech Co., Ltd.) under the following thermocycling conditions: $95^{\circ} \mathrm{C}$ for $15 \mathrm{~min}$, followed by 40 cycles of $95^{\circ} \mathrm{C}$ for $10 \mathrm{sec}$ and $60^{\circ} \mathrm{C}$ for $30 \mathrm{sec}$. The expression levels were quantified using the $2^{-\Delta \Delta \mathrm{Cq}}$ method with GAPDH and U6 as the internal controls (17). The reverse primer for miR-3907 was universal (included in the miRcute Plus miRNA qPCR kit), and the other primer sequences are displayed in Table I.

Immunohistochemical staining. The 18 SGC and 6 control FFPE samples were deparaffinized using xylene for $10 \mathrm{~min}$ three times at room temperature. (Kermel Biotech Co.,Ltd.) and dehydrated in gradient ethanol. Sodium citrate ( $\mathrm{pH}$ 6; Beijing Zhongshan Jinqiao Biotech Co., Ltd.) was used for antigen repair for $20 \mathrm{~min}$. After treating with $3 \% \mathrm{H}_{2} \mathrm{O}_{2}$ (SP9000 Kit; Beijing Zhongshan Jinqiao Biotech Co., Ltd.), washing in PBS and blocking for $30 \mathrm{~min}$ with goat serum (SP9000 Kit; Beijing Zhongshan Jinqiao Biotech Co., Ltd.) at room temperature, the sections were incubated with polyclonal rabbit anti-human THBS1 antibody (1:500; Affinity Biosciences, Ltd.) at $4^{\circ} \mathrm{C}$ overnight. The following day, the sections were incubated with a polyclonal rabbit anti-human THBS1 secondary antibody (1:500; Affinity Bioscience, Ltd.) for $30 \mathrm{~min}$ at room temperature, and then stained with DAB (Zhongshan Jinqiao Biotech Co., Ltd.). Finally, sections were stained for $20 \mathrm{sec}$ with hematoxylin at room temperature, dehydrated and sealed. ImageJ 6.0 software (National Institutes of Health) was used for image analysis.

Cell transfection. miR-3907 mimics, miR-3907 inhibitor, small interfering RNA (siRNA)-THBS1 and the corresponding negative controls (NCs) were synthesized and purchased from Shanghai GenePharm Co., Ltd. According to the manufacturer's instructions, different volumes of diethylprocarbonate water were added to each mimic, inhibitor, siRNA and corresponding NC to ensure a concentration of $20 \mu \mathrm{M}$ per tube. The transfections were performed using Lipofectamine ${ }^{\circledR} 3000$ (Invitrogen, Thermo Fisher Scientific, Inc.) according to the manufacturer's instructions. A total of $3.75 \mu \mathrm{l}$ Lipofectamine and $125 \mu \mathrm{l}$ RPMI-1640 medium were mixed to create Mix 1. For Mix 2, $125 \mu$ R RMI-1640 medium, $10 \mu \mathrm{l}$ P3000 and $5 \mu \mathrm{l}$ DNA plasmid were mixed. Mix 1 and Mix 2 were then combined, incubated for $10 \mathrm{~min}$ in the dark at room temperature, and then added into 6-well 
Table I. Primer sequences.

Gene

Forward (5' to $\left.3^{\prime}\right)$

Reverse (5' to $\left.3^{\prime}\right)$

$\begin{array}{ll}\text { microRNA-3907 } & \text { AGGTGCTCCAGGCTGGCTCACA } \\ \text { U6 } & \text { CTCGCTTCGGCAGCACA } \\ \text { Thrombospondin } 1 & \text { AGACTCCGCATCGCAAAGG } \\ \text { GAPDH } & \text { GATGCTGGCGCTGAGTACG }\end{array}$

The reverse primer for miR-3907 was universal, and included in the miRcute Plus miRNA qPCR kit.

Table II. Transfectant sequences.

\begin{tabular}{lc}
\hline Gene & \multicolumn{1}{c}{ Sequence (5' to 3') } \\
\hline $\begin{array}{l}\text { miR-3907mimics } \\
\text { Sense }\end{array}$ & \\
Antisense & AGGUGCUCCAGGCUGGCUCACA \\
miR-3907mimics-NC & UGAGCCAGCCUGGAGCACCUUU \\
Sense & UUCUCCGAACGUGUCACGUTT \\
Antisense & ACGUGACACGUUCGGAGAATT \\
miR-3907 inhibitor & UGUGAGCCAGCCUGGAGCACCU \\
miR-3907 & CAGUACUUUUGUGUAGUACAA \\
inhibitor-NC & \\
siRNA-THBS1 & \\
Sense & GCGUGUUUGACAUCUUUGATT \\
Antisense & UCAAAGAUGUCAAACACGCTT \\
siRNA-THBS1 NC & \\
Sense & UUCUCCGAACGUGUCACGUTT \\
Antisense & ACGUGACACGUUCGGAGAATT
\end{tabular}

miR, microRNA; siRNA, small interfering RNA; THBS1, thrombospondin $1 ; \mathrm{NC}$, negative control.

plates containing SGC eyelid primary cells at room temperature. CCK-8 and wound healing experiments were performed on the second day after transfection. RNA was extracted from 48 to $72 \mathrm{~h}$ after transfection, and protein was extracted from 72 to $96 \mathrm{~h}$ after transfection. THBS1 lentivirus vector was synthesized by Shanghai Obio Biotechnology Co., Ltd. (pSLenti-SFH-EGFP-P2A-Puro-CMV-THBS1-3xFLAG-WPRE). The third lentivirus generation system was used and the interim 293T cell line (ATCC) was used. The quantity of lentiviral plasmid used for transfection was $10 \mu \mathrm{g}$ in a $10 \mathrm{~cm}$ cell culture dish, and the ratio of the lentiviral plasmid: packaging vector: envelope was 0.25:1:0.75. After transfection for $48 \mathrm{~h}$ at room temperature, the supernatant was collected and the medium was changed. The supernatant was collected again after $72 \mathrm{~h}$ of transfection and merged with the medium collected at $48 \mathrm{~h}$. On reaching 30-40\% confluency, the SGC cells were transfected at room temperature with plasmids at an MOI of 40. The medium was replaced within $24 \mathrm{~h}$ and the fluorescence signal was observed under a fluorescence microscope after $72 \mathrm{~h}$ at room tamperature. Lentivirus plasmid was transfected successfully and subsequent experiments were carried out $72 \mathrm{~h}$ after transfection. The sequences of mimics, inhibitor, siRNA-THBS1 and NC are displayed in Table II.

miR-3907 target gene prediction and dual-luciferase reporter assay. The TargetScan 7.2 (18) and miRDB databases (19) were used to predict the target genes of miR-3907. The wild-type pGL3-THBS1-3' untranslated region (UTR) (WT-THBS1) and mutant pGL3-THBS1-3'UTR (MUT-THBS1) expression vectors were constructed by Shanghai GenePharma Co., Ltd. 293T cells were co-transfected with miR-3907 mimics and mimics-NC with the WT-THBS1 and MUT-THBS1 vectors, using Lipofectamine ${ }^{\circledR} 3000$. After transfection for $48 \mathrm{~h}$, the relative firefly luciferase activity (the luciferase activity ratio of Renilla luciferase activity to firefly luciferase activity) was determined using the Dual-Luciferase Reporter Assay System (Promega Corporation).

Western blotting. Total cellular protein was extracted using RIPA buffer containing 1\% protease inhibitor (Beijing Solarbio Science \& Technology Co., Ltd.), and a BCA kit was used to determine the protein concentration (Beijing Solarbio Science \& Technology Co., Ltd.). Protein samples were mixed with $5 \mathrm{X}$ loading buffer (Beijing Solarbio Science \& Technology Co., Ltd.) and denatured at $99^{\circ} \mathrm{C}$ for $10 \mathrm{~min}$. Then, $40 \mu \mathrm{g}$ protein sample per lane was loaded and separated via $10 \%$ SDS-PAGE, and then transferred to a PVDF membrane (Bio-Rad Laboratories, Inc.). The membrane was blocked with $5 \%$ skim milk at room temperature for $2 \mathrm{~h}$, and then incubated with primary antibodies against, THBS1 (1:1,000; cat. no. DF6848; Affinity Biosciences, Ltd.) and polyclonal mouse anti-human GAPDH (1:1,000; cat. no. T0004; Affinity Biosciences, Ltd.) at $4^{\circ} \mathrm{C}$ overnight with gentle shaking. The following day, after washing with $1 \mathrm{X}$ TBS with $0.05 \%$ Tween-20 (TBST; Gibco; Thermo Fisher Scientific, Inc.), the membrane was incubated with the anti-mouse IgG [heavy chain $(\mathrm{H})+$ light chain $(\mathrm{L})$ ] biotinylated secondary antibody (1:2,000; cat. no. 14709; Cell Signaling Technology, Inc.) or anti-rabbit $\operatorname{IgG}(\mathrm{H}+\mathrm{L})$ biotinylated secondary antibody (1:2,000; cat. no. 14708; Cell Signaling Technology, Inc.) for $2 \mathrm{~h}$ at room temperature. After further washing with $1 \mathrm{X}$ TBST, protein bands were visualized using a Omni-EC ${ }^{\text {TM }}$ Pico Light Chemiluminescent Kit (Shanghai Epizyme Biotech Co., Ltd.). Images were captured using the Tanon 480 photoluminescence imaging system (Tanon Science and Technology Co., Ltd.). ImageJ 6.0 software (National Institutes of Health) was used for semi-quantification analysis. 

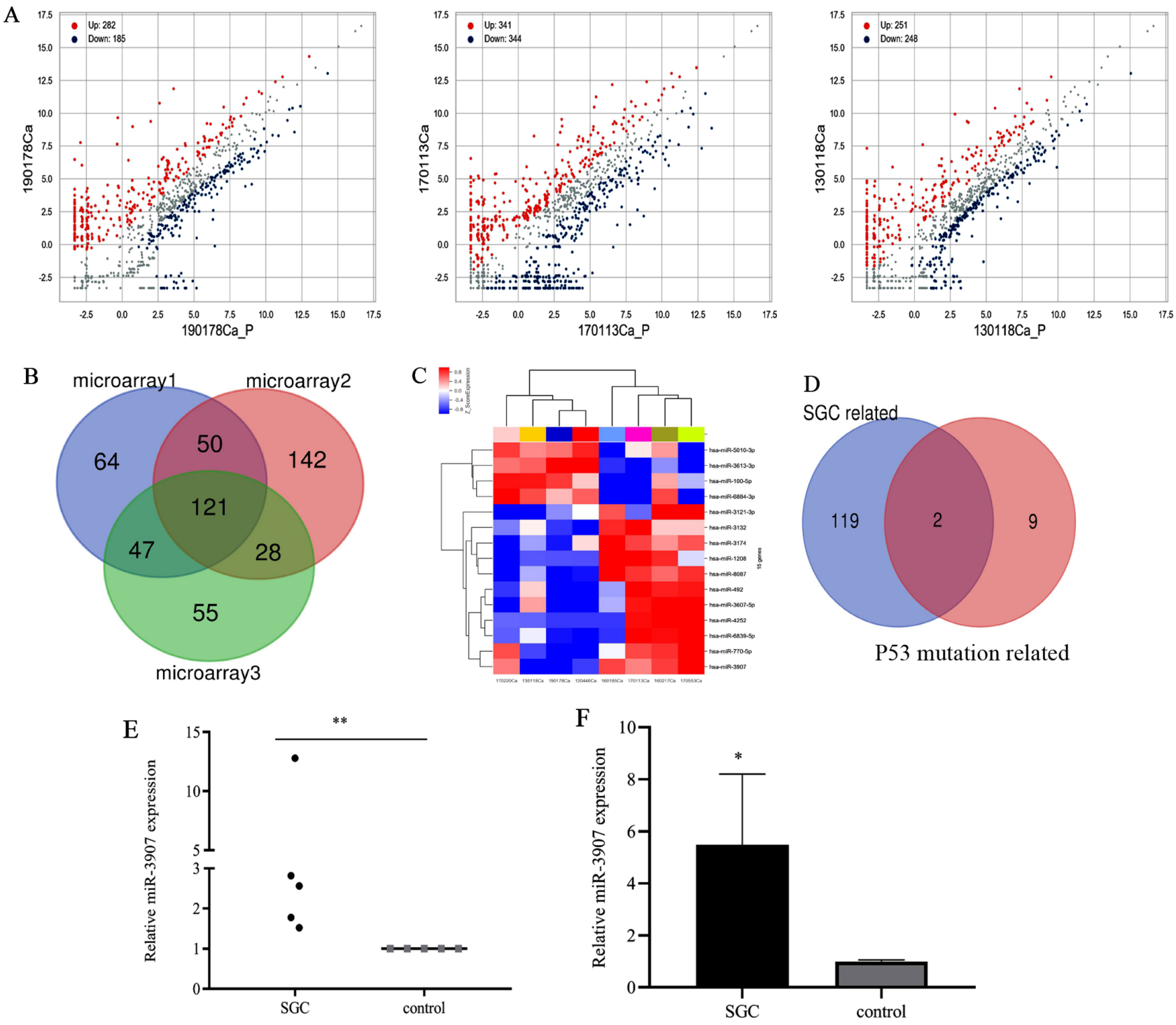

Figure 1. Screening and expression of miR-3907 in tissues and cells of patients with SGC. (A) Microarray of differentially expressed miRNAs in SGC (B) Venn diagram of differentially upregulated miRNAs in 3 SGC tissues. (C) Heat map of differentially expressed miRNAs in SGC tissues containing a P53 mutation. (D) Venn diagram of differentially upregulated miRNAs between two sets of miRNA microarray data. (E) Relative expression level of miR-3907 in SGC tissues was higher than that in para-carcinoma tissues. (F) Relative expression levels of miR-3907 in SGC cells compared with normal SG cells. "P<0.05 and ${ }^{* *} \mathrm{P}<0.01$. miRNA/miR, microRNA; SGC, sebaceous gland cancer.

Cell Counting Kit $8(C C K-8)$ assay. After digestion and counting, SGC cells were seeded at $5 \times 10^{3}$ cells/well in 96 -well plates. Then, $10 \mu \mathrm{l}$ CCK-8 solution was added to each well at different time points, and the plates were incubated for $3 \mathrm{~h}$. The absorbance values at $450 \mathrm{~nm}$ were determined using a plate reader (Infinite M200 Pro; Tecan Group, Ltd.).

Wound-healing assay. A 6-well plate was inoculated with SGC cell suspension. After transfection and when the cells reached 70-90\% confluence, a $100-\mu 1$ pipette tip was used to create a scratch in each monolayer. After rinsing with PBS, 2 ml 1\% low concentration serum medium was added, and the plate was returned to the incubator. Images were captured $24 \mathrm{~h}$ post-wounding; the wound area was observed at 0 and $24 \mathrm{~h}$ under an inverted microscope. The relative migration distance to the wound area was quantified and the migration wound healing rate was calculated using Image J software 6.0. Migration wound healing rate $=(0 \mathrm{~h}$ wound area-24 h wound area)/0 h wound area $\times 100 \%$.
Statistical analysis. SPSS 23.0 statistical software (IBM Corp) was used for data analysis. Two-tailed unpaired Student's t-test, one-way ANOVA followed by Dunnett's multiple comparisons test were performed. The experimental results are presented as the mean $\pm \mathrm{SD}$, and $\mathrm{P}<0.05$ was considered to indicate a statistically significant difference.

\section{Results}

Upregulation of miR-3907 in SGC tissues and primary cells. Differentially expressed miRNAs in three SGC and para-carcinoma control tissues were analyzed using a miRNA microarray, in which 467, 685 and 499 differentially expressed miRNAs were respectively identified (Fig. 1A). The intersection of these miRNAs revealed that there were 121 differentially upregulated miRNAs (Fig. 1B). With regards to the differential miRNAs in the four SGC samples with P53 mutation, and the four SGC tissues without P53 mutation, microarray 

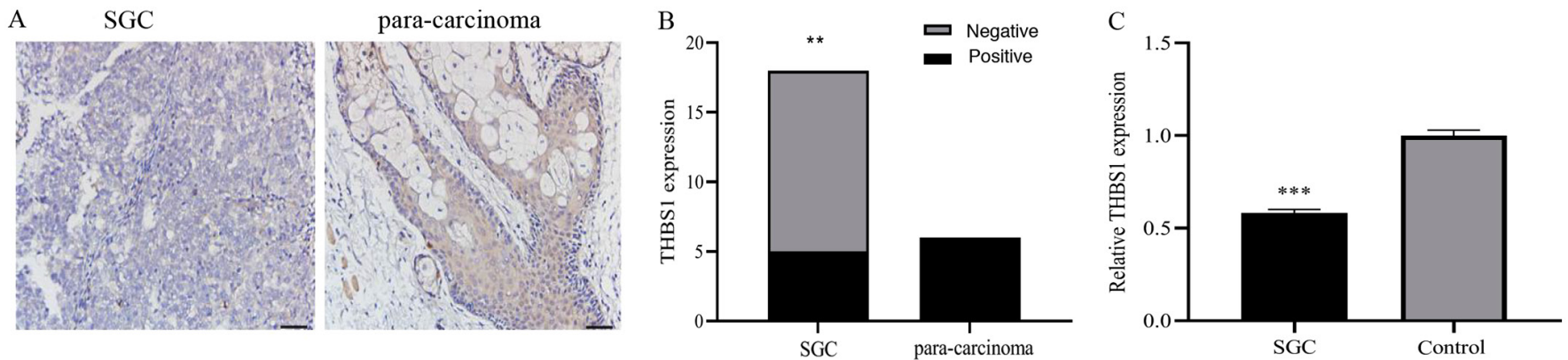

Figure 2. THBS1 expression levels in SGC tissues and cells. (A) Immunohistochemical staining of THBS1 in SGC and para-carcinoma tissues. Expression of THBS1 was negative in SGC tissues, but positive in para-carcinoma tissues. Scale bar, $50 \mu \mathrm{m}$. (B) Positive expression rate of THBS1 in eyelid SGC and para-carcinoma formalin-fixed paraffin-embedded samples. (C) Relative mRNA expression levels of THBS1 in SGC cells were lower than those in normal SG cells. ${ }^{* *} \mathrm{P}<0.01$ and ${ }^{* * * *} \mathrm{P}<0.001$. SGC, sebaceous gland cancer; THBS1, thrombospondin 1.

revealed 15 differential miRNAs, of which 11 were upregulated (Fig. 1C). There were only two miRNAs (miR-3907 and miR-3132) that overlapped between the 121 and 11 differentially upregulated miRNAs (Fig. 1D). Using RT-qPCR, the expression level of miR-3907 was found to be significantly higher in SGC tissues than in para-carcinoma tissues (P<0.01; Fig. 1E). Next, the expression levels of miR-3907 in SGC primary cells were determined, and identified to also be significantly upregulated compared with those in healthy SG primary cells $(\mathrm{P}<0.05$; Fig. 1F). Therefore, miR-3907 was selected and was demonstrated to be upregulated in eyelid SGC.

THBS1 expression in SGC tissues. Immunohistochemical staining was used to evaluate the protein expression level of THBS1 in SGC tissues, which was found to be decreased compared to that of para-carcinoma tissues (Fig. 2A). Furthermore, the THBS1-positive expression rate $(5 / 18 ; 27.8 \%)$ in SGC tissues was lower than in the control tissues $(6 / 6 ; 100 \%$; Fig. 2B; P<0.01. Next, RT-qPCR was used to assess THBS1 mRNA expression in SGC cells, which was found to be significantly downregulated in SGC cells compared with normal SG cells (Fig. 2C; $\mathrm{P}<0.001$ ).Therefore, the results indicated that THBS1 expression levels were downregulated in eyelid SGC.

Promotive effects of miR-3907 in SGC cells. To investigate whether miR-3907 affects SGC cell tumorigenesis, miR-3907 was overexpressed or knocked down by transfection with miR-3907 mimics or miR-3907 inhibitor, respectively, and the expression level was analyzed using RT-qPCR. The RT-qPCR results demonstrated successful miR-3907 mimic transfection $(\mathrm{P}<0.001$; Fig. 3A). The proliferative and migratory abilities of SGC cells were determined using CCK-8 and wound-healing assays, the results indicated that compared with the control group, cellular proliferation was significantly increased after miR-3907 overexpression ( $\mathrm{P}<0.001$; Fig. 3B), migration ability was also increased $(\mathrm{P}<0.01$; Fig. $3 \mathrm{C})$. The RT-qPCR results demonstrated that miR-3907 was knocked down successfully $(\mathrm{P}<0.01$; Fig. 3D). The CCK-8 and wound-healing results showed that compared with the control group, proliferation ability was decreased following transfection with the miR-3907 inhibitor $(\mathrm{P}<0.05$; Fig. 3E). Migration ability also decreased after transfection with the miR-3907 inhibitor $(\mathrm{P}<0.001$; Fig. 3F). Therefore, miR-3907 may play a carcinogenic role in eyelid SGCs by affecting proliferation and migration.
miR-3907 negatively regulates THBS1 expression. To investigate how miR-3907 affects SGC cell proliferation and migration, bioinformatics databases were used to predict the putative target of miR-3907. The prediction results revealed complementary binding sites between miR-3907 and the 3'UTR of THBS1 (Fig. 4A). Next, the binding association between miR-3907 and THBS1 was investigated. The dual-luciferase reporter assay results demonstrated that the luciferase activity of the miR-3907mimics + WT-THBS1 group was significantly lowerthan that of the mimics-NC+WT-THBS1 group $(\mathrm{P}<0.001)$, while the activity of the miR-3907mimics + MUT-THBS1 group was not significantly different from that of the mimics-NC + MUT-THBS1 group (Fig. 4B). Furthermore, the RT-qPCR and western blotting results suggested that THBS1 mRNA and protein expression were significantly downregulated in the miR-3907 mimics group compared with the mimics-NC group (Fig. 4C and D), which further indicated the regulatory relationship between miR-3907 and THBS1. The results suggested that miR-3907 negatively regulated THBS1 expression.

Inhibitory effects of THBS1 in SGC cells. To investigate whether THBS1 affects SGC cell tumorigenesis, THBS1 was overexpressed or knocked down by transfecting cells with THBS1 lentivirus vector or siRNA-THBS1, respectively, and THBS1 expression was analyzed and observed using RT-qPCR and fluorescence microscopy, respectively. RT-qPCR (Fig. 5A; $\mathrm{P}<0.001)$ demonstrated successful siRNA transfection. CCK-8 and wound-healing assays indicated that, compared with the control group, the proliferative ability of the siRNA-THBS1 group were significantly increased (Fig. 5B; $\mathrm{P}<0.05$ ) and the migratory ability of the siRNA-THBS1 group increased (Fig. 5C; P<0.001). RT-qPCR (Fig. 5D; P<0.001)and fluorescence microscopy (Fig. 5E) demonstrated successful lentiviral vector transfection. While in the THBS1 overexpression group, these properties were significantly inhibited, including proliferation (Fig. 5F; P<0.05) and migration (Fig. 5G; P<0.01). These observations suggested that THBS1 serves an important inhibitory role in eyelid SGC by inhibiting the proliferation and migration of SGC cells.

Oncogenic effects of miR-3907 exerted by targeting THBSI in SGC cells. To investigate whether miR-3907 expression is associated with SGC through THBS1, SGC cells 

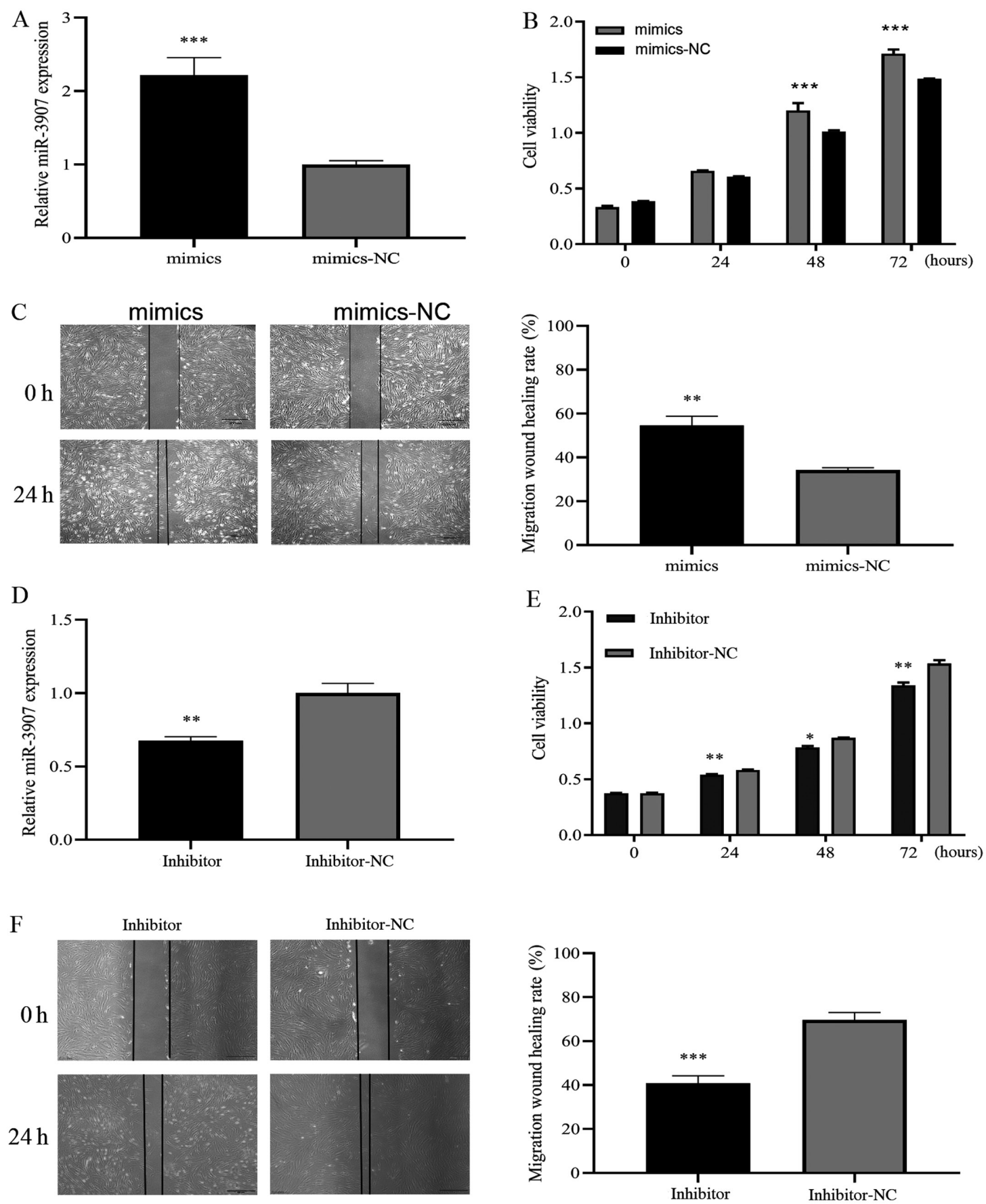

Figure 3. Promotive effects of miR-3907 on SGC cells proliferation and migration. (A) SGC cells were transfected with miR-3907 mimics, and miR-3907 expression level was determined by reverse transcription-quantitative PCR. Results of (B) CCK-8 assay and (C) wound-healing assays following transfection of SGC cells with miR-3907 mimics. CCK-8 and wounding-healing assays showed that miR-3907 mimics promoted the proliferation and migration of SGC cells. (D) SGC cells were transfected with miR-3907 inhibitor, and miR-3907 expression level was determined using reverse transcription-quantitative PCR. Results of (E) CCK-8 and (F) wound-healing assays after SGC cell transfection with an miR-3907 inhibitor. The assays showed that the miR-3907 inhibitor decreased the proliferation and migration of SGC cells. Scale bar, $500 \mu \mathrm{m} .{ }^{*} \mathrm{P}<0.05,{ }^{* *} \mathrm{P}<0.01$ and ${ }^{* * * *} \mathrm{P}<0.001$. SGC, sebaceous gland cancer; miR, microRNA; CCK-8, Cell Counting Kit-8; NC, negative control.

were co-transfected with miR-3907 mimics and THBS1, or inhibitor and siRNA-THBS1. CCK8 and wound-healing assay results indicated that compared with the miR-3907 mimics-NC group, overexpression of miR-3907 increased proliferation and migration. However, when cells were co-transfected with both miR-3907 and THBS1, these properties including proliferation (Fig. 6A; $\mathrm{P}<0.01$ ) and migration (Fig. 6B; $\mathrm{P}<0.001$ ) were decreased compared with the miR-3907 mimics group. Similar trends were also observed when cells were co-transfected with the inhibitor and siRNA-THBS1. For instance, when cells were transfected with the miR-3907 inhibitor, cell proliferation and migration were decreased compared with the inhibitor-NC group. However, when both miR-3907 and THBS1 were silenced, there was a significant increase in proliferation (Fig. 6C; $\mathrm{P}<0.05$ )and migration (Fig. 6D; $\mathrm{P}<0.05$ ) compared with the inhibitor group. Therefore, the results indicated that miR-3907 mediated eyelid SGC via targeted regulation 

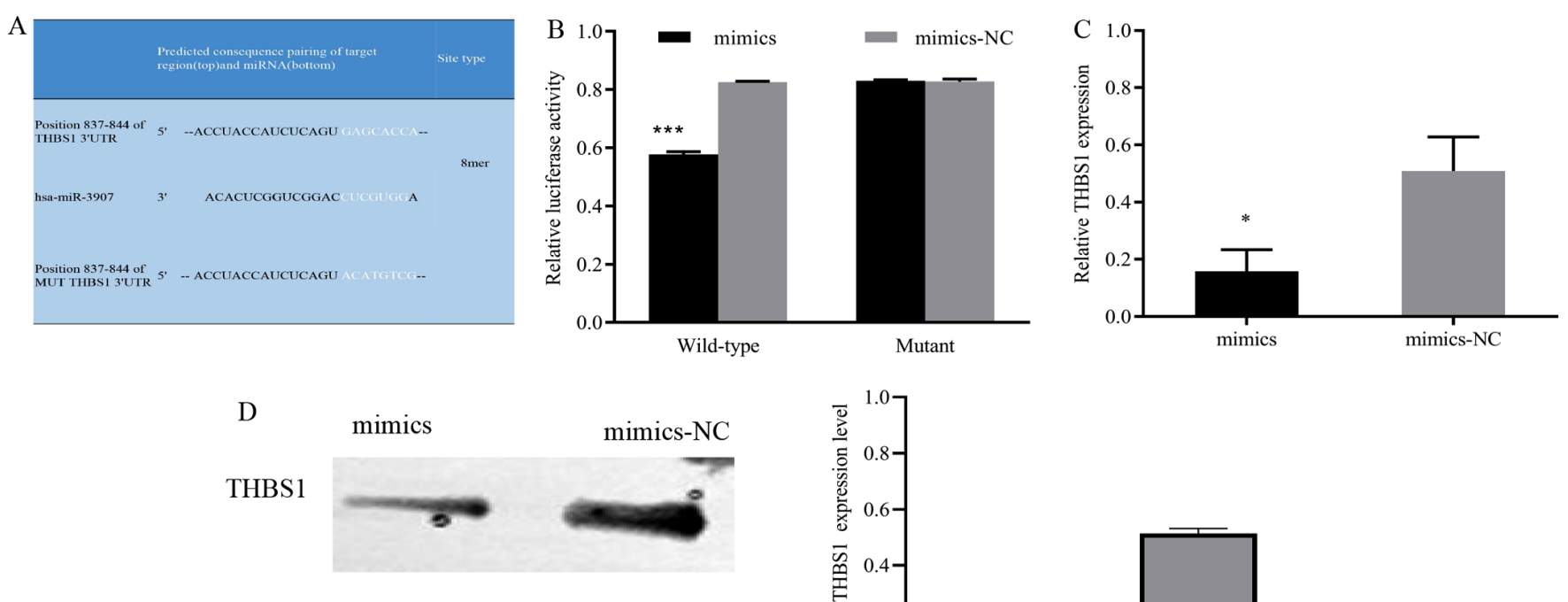

GAPDH
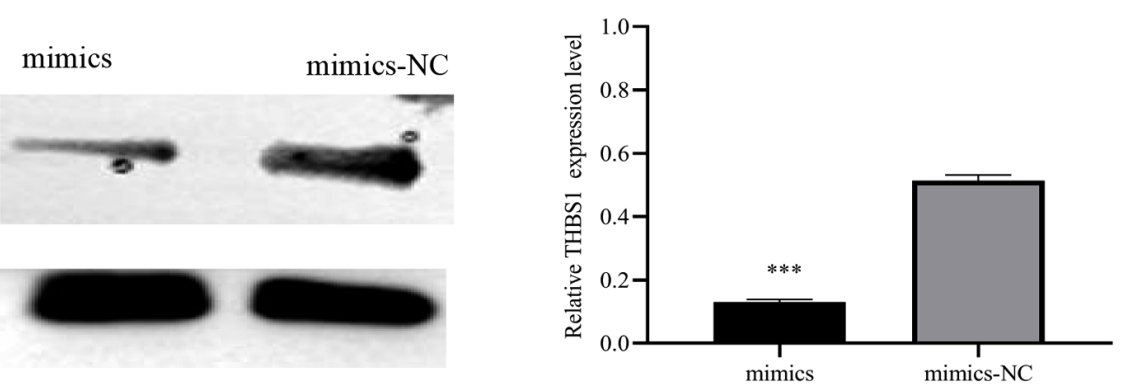

Figure 4. miR-3907 negatively regulates THBS1 expression. (A) Predicted binding sites for miR-3907 within the wild-type and mutant 3'UTR of THBS1. (B) Quantitative analysis of the relative luciferase activity of cells with reporter vectors containing the wild type or mutant THBS1 3'UTR, following miR-3907 co-transfection. (C) Reverse transcription-quantitative PCR and (D) western blotting with semi-quantification analysis revealed decreased THBS1 expression following miR-3907 mimics transfection. ${ }^{*} \mathrm{P}<0.05$ and ${ }^{* * *} \mathrm{P}<0.001$. miR, microRNA; THBS1, thrombospondin 1; UTR, untranslated region; NC, negative control.
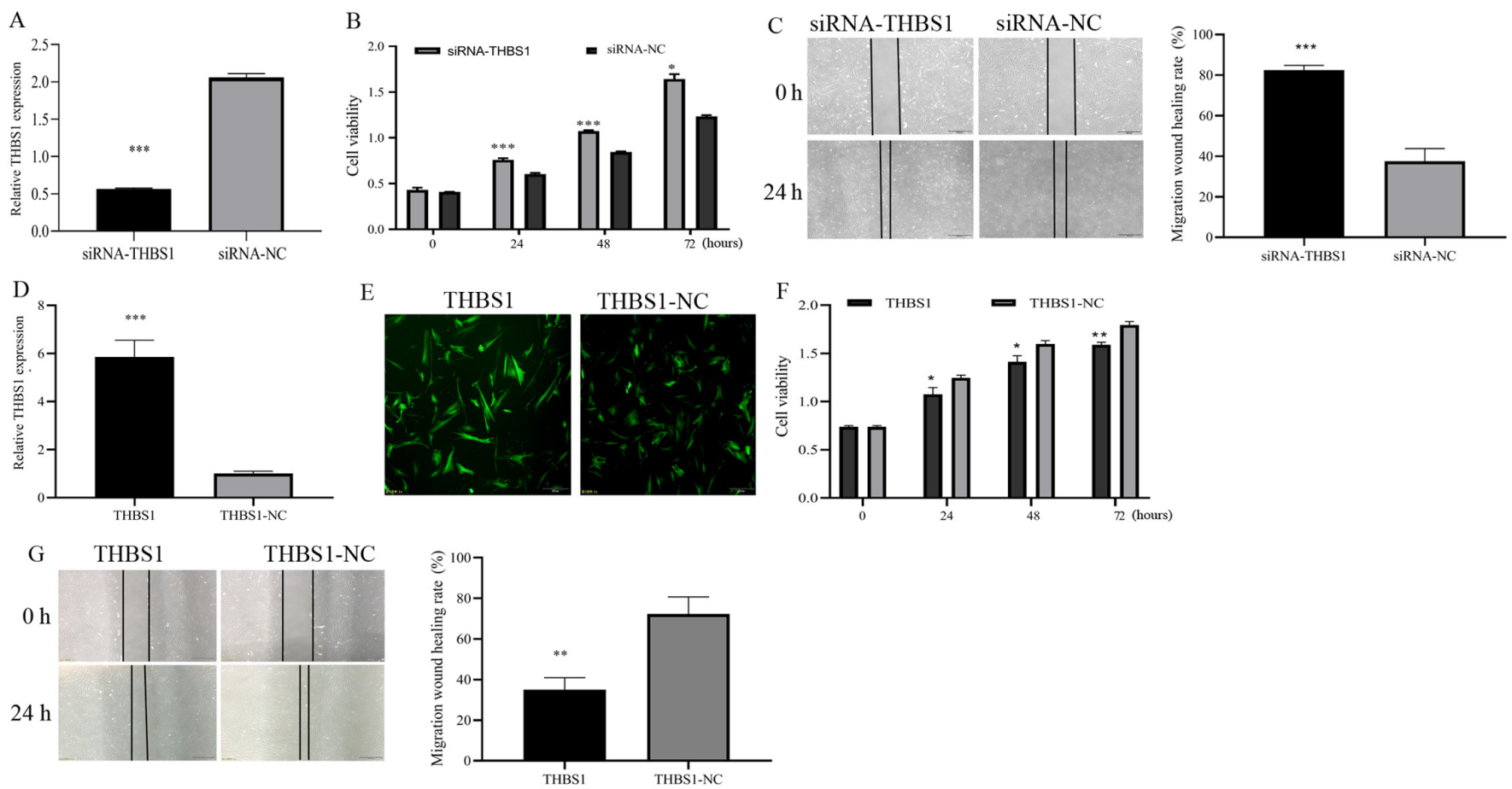

Figure 5. Inhibitory effects of THBS1 on SGC cell proliferation and migration. (A) THBS1-knockdown was induced by transfecting SGC cells with siRNA-THBS1. THBS1 expression level was determined by reverse transcription-quantitative PCR. Results of (B) CCK-8 and (C) wound-healing assays after transfection with siRNA-THBS1; siRNA-THBS1 increased the proliferation and migration of SGC cells. (D) THBS1 overexpression was induced by transfecting lentiviral vectors; THBS1 expression level was determined by reverse transcription-quantitative PCR. (E) Fluorescence assay results revealed that the THBS1 lentivirus vector was successfully transfected. (F) CCK-8 and (G) wound-healing assays results after transfection with THBS1 lentivirus revealed that THBS1 decreased the proliferation and migration of SGC cells. Scale bar, $500 \mu \mathrm{m}$. "P<0.05, ${ }^{* *} \mathrm{P}<0.01$ and ${ }^{* * * *} \mathrm{P}<0.001$. SGC, sebaceous gland cancer; siRNA, small interfering RNA; CCK-8, Cell Counting Kit-8; THBS1, thrombospondin 1; NC, negative control.

of THBS1. Overall, miR-3907 may serve as an oncogene in SGC of the eyelid, and THBS1 may be a tumor suppressor gene. miR-3907 targeted the THBS1 3'UTR and regulated THBS1 expression levels. Furthermore, co-transfection was demonstrated to reverse the single effect of miR-3907 mimics or inhibitors. Thus, it was concluded that miR-3907 mediated the proliferation and migration of eyelid SGC via targeting THBS1. 

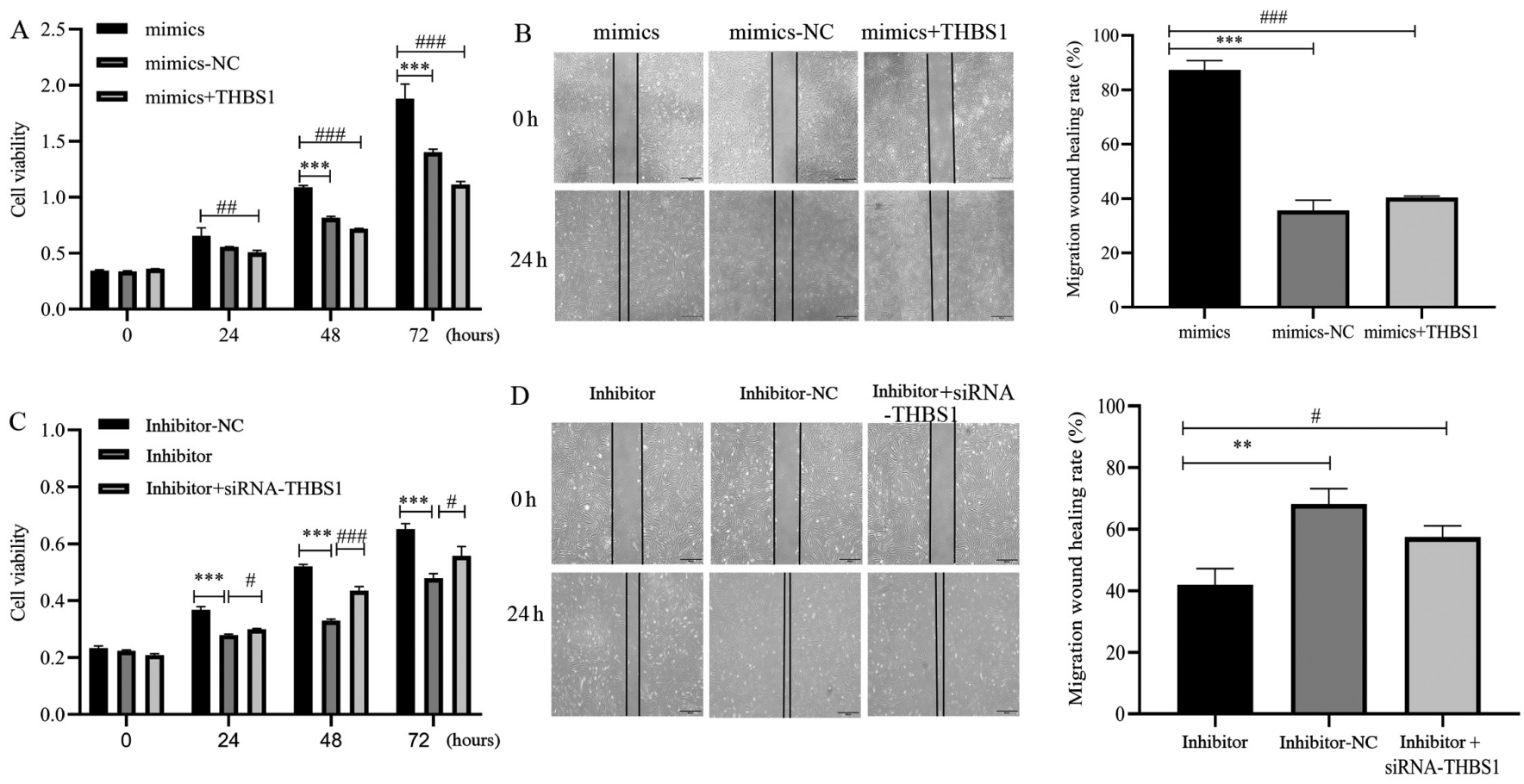

Figure 6. Oncogenic effects of miR-3907 on SGC cells via THBS1 targeting. Cellular (A) proliferation and (B) migration were evaluated by CCK-8 and wound-healing assays after SGC cell transfection with mimic-NC, miR-3907 mimics, or co-transfection with miR-3907 mimics + THBS1. Cellular (C) proliferation and (D) migration were evaluated by CCK-8 and wound-healing experiments, respectively, in SGC cells transfected with inhibitor-NC, miR-3907 inhibitor, or co-transfected with miR-3907 inhibitor + siRNA-THBS1. Scale bar, $500 \mu \mathrm{m}$. ${ }^{* *} \mathrm{P}<0.01$ and ${ }^{* * * *} \mathrm{P}<0.001 ;{ }^{*} \mathrm{P}<0.05,{ }^{\# \#} \mathrm{P}<0.01$ and ${ }^{\# \# \#} \mathrm{P}<0.001$. SGC, sebaceous gland cancer; siRNA, small interfering RNA; CCK-8, Cell Counting Kit-8; THBS1, thrombospondin 1; miR, microRNA; NC, negative control.

\section{Discussion}

SGC of the eyelid originates from the SG of the human eyelid, especially the upper eyelid (20), and is a common eyelid malignancy. The disease usually occurs in the elderly, is more frequently observed in women (2), and patients are more prone to recurrence and metastasis, leading to a poorer prognosis. Patients in early disease stages may have painless hard nodules in the eyelid, and as the disease progresses, may experience eyelid swelling and thickening, irregular lobulated or cauliflower yellow tissues on the conjunctival surface, and even local rupture of the tumor $(5,21)$. However, early SGC can easily be confused with benign eyelid tumors (for example, chalazions), and as such, should be diagnosed and treated as soon as possible $(22,23)$. At present, the primary treatment options for SGC are surgical resection or combined chemoradiotherapy. However, due to early misdiagnosis and mistreatment, local recurrence, invasion and distant metastasis, as well as insensitivity to chemoradiotherapy, the therapeutic effect is not always achieved $(24,25)$. Therefore, understanding SGC pathogenesis from the molecular level is important for the development of targeted therapy, and to improve patient prognosis.

As non-coding RNAs, miRNAs can form a complex regulatory network and play an important role in the occurrence and development of various malignant tumors (26). In addition, due to their tissue-specific and highly conserved characteristics, the abnormal expression of miRNAs may be used as an important marker for evaluating cancer progression and patient prognosis, including in $\operatorname{SGC}(9,10,27,28)$. Bhardwaj et al (10) reported that the expression levels of
miR-200c and miR-141 were lower in 86 and $67 \%$ cases of eyelid SGC tissues compared with para-carcinoma control tissues. Moreover, Bladen et al (27) conducted a sequencing study on different SGC subtypes (nodules and pagetoid) and normal SG tissues to investigate differential miRNA expression. Hirano et al (9) performed a small RNA-sequencing analysis to identify differentially expressed miRNAs between SGC and sebaceous adenoma samples, as well as bioinformatics analyses to reveal biological functions, related pathways and molecular interaction networks. Thus, these methods may ultimately improve our understanding of the role of differentially expressed miRNAs in tumor progression.

P53, a tumor suppressor gene, loses the ability to regulate cellular proliferation, apoptosis and DNA repair after mutation. P53 mutation can be found in a variety of malignant tumors, including SGC; in fact, a number of studies have reported that $\mathrm{P} 53$ is the most common mutation in $\operatorname{SGC}(29,30)$. The protein expression levels of P53 have also been shown to be significantly increased after mutation compared with patients with SGC without P53 mutation. Furthermore, patients with a P53 mutation were more prone to local recurrence, and their tumor cells were poorly differentiated (31). On this basis, in addition to conducting an miRNA microarray between SGC and para-carcinoma tissues, an miRNA microarray between four SGC samples containing P53 mutation and four SGC tissues samples without P53 mutation was conducted in the present study. The overlap between the two sets of differentially upregulated miRNAs generated miR-3907 and miR-3132 only; on review of the current literature, no studies on the association between miR-3132 and malignant tumors were found, and its role in other malignant tumors is unclear. Therefore, based 
on previous studies and fold change analyses, miR-3907 was finally selected for further investigation in the current study.

There have been a few studies to investigate miR-3907, and these have reported that miR-3907 has underlying diagnostic value for several diseases, as it has been reported to be dysregulated in lung cancer and kidney disease. For instance, the expression level of miR-3907 was increased in polycystic kidney disease, and the sensitivity and specificity of the receiver operating characteristic curve were high, suggesting that miR-3907 may be used to predict the progress of polycystic kidney disease (7). Furthermore, compared with the control tissues, the expression level of miR-3907 was higher in lung cancer tissues, and may be a potential early biomarker of the malignant transformation of bronchial epithelial cells (11).

However, to the best of our knowledge, there have been no previous reports on the role of miR-3907 in SGC, and the present study may be the first to demonstrate such a role. Herein, the level of miR-3907 was found to be increased in SGC tissues and cells compared with that observed in the control group. To further confirm the role of miR-3907, miR-3907 mimics promoted a significant increase in the proliferation and migration abilities of SGC cells, while the miR-3907 inhibitor had the opposite effect, suggesting that miR-3907 may play an important role as an oncogene gene in SGC, which was similar to the reported cancer-promoting effect of miR-3907 in lung cancer tissues (11).

The mechanisms underlying the promotive effects of miR-3907 on cellular proliferation and migration were investigated by identifying its target gene, THBS1, using bioinformatics analysis and a dual-luciferase reporter assay. The data indicated that miR-3907 may promote SGC occurrence and progression by directly binding and negatively regulating the expression of THBS1.

The platelet thrombin protein THBS1, expressed in the cellular membrane, endoplasmic reticulum and extracellular matrix, plays an important role in platelet aggregation, angiogenesis and the occurrence and development of a variety of malignant tumors $(32,33)$. The expression level and roles of THBS1 vary in different tumor types, where it can play either a stimulatory and inhibitory role. For instance, von Hippel-Lindau protein-mediated THBS1 decreased the migration of clear cell renal carcinoma cell lines (34). Furthermore, the expression level of THBS1 was positively correlated with overall survival in non-small cell lung cancer (35). However, other studies have shown that THBS1 is highly expressed in oral squamous cell carcinoma, where it facilitates cellular invasiveness and migration (36). Moreover, exosomal THBS1 promoted the migration of breast cancer cells by disrupting the intercellular integrity of endothelial cells (37). In the present study, the expression level of THBS1 was downregulated in SGC tissues and cells. THBS1-knockdown was also found to promote cellular proliferation and migration, while THBS1 overexpression inhibited these properties. Therefore, THBS1 was suggested to possess potent activity against SCG, and overexpression of THBS1 may be a means of suppressing SGC development.

The present study also indicated that THBS1 was targeted and negatively regulated by miR-3907. To further verify the association between miR-3907 SGC by targeting THBS1, SGC cells were co-transfected with THBS1 and miR-3907 mimics,
miR-3907 inhibitor and siRNA-THBS1. THBS1 and miR-3907 mimic co-transfection were found to reverse the promotive effect of miR-3907 mimics on SGC cells, and siRNA-THBS1 and mir-3907 inhibitor co-transfection reversed the inhibitory effect of miR-3907 inhibitor. Therefore, miR-3907 may regulate the biological functions of SGC cells, such as proliferation and migration, by targeting THBS1.

In conclusion, based on the results of miRNA microarray and molecular biology techniques, the present study initially demonstrated the role and possible regulatory mechanism of miR-3907 in SGC. The mechanisms underlying the miR-3907/THBS1 axis may lead to the development of important diagnostic and treatment strategies, such as novel targeted drugs, to improve patient prognosis. However, due to the small size of human eyelids, the availability of SGC tissues samples was limited, and the sample size for microarray and immunohistochemical staining was small, which is a limitation of the present study. Further investigations with an increased number of samples are required to validate these results, and to elucidate the roles of the miR-3907/THBS1 axis in SGC.

\section{Acknowledgements}

Not applicable.

\section{Funding}

The present study was supported by grants from The National Natural Science Foundation of China (grant no. 81570872 and 81800825), The Tianjin Municipal Health Commission (grant no. MS20025) and The Tianjin Key Clinical Discipline Construction Project (grant no. TJLCZDXKT006 and TJLCZDXKQ024).

\section{Availability of data and materials}

The datasets used and/or analyzed during the present study are available from the corresponding author upon reasonable request. The microarray datasets generated and/or analyzed during the present study are available in the GEO database (accession no. GSE182876).

\section{Authors' contributions}

All the authors conducted the experiments, and all the authors confirmed the authenticity of all raw data. CZ, LZ, XL and MJ conceived the study and drafted the initial manuscript. QT and FX analyzed and interpretated the data. TL, LD and YH revised the manuscript for important intellectual content. LZ, TL and YH collected the clinical data, made the final diagnoses and performed the patient surgeries. All authors have read and approved the final manuscript.

\section{Ethics approval and consent to participate}

The present study was approved by The Institutional of Human Research Ethics Committee of the Tianjin Medical University Eye Hospital [approval no. 2020KY(L)-2], and all procedures were performed in accordance with the Declaration of Helsinki. All patients provided written informed consent. 


\section{Patient consent for publication}

Not applicable.

\section{Competing interests}

The authors declare that they have no competing interests.

\section{References}

1. Cook BJ and Bartley GB: Treatment options and future prospects for the management of eyelid malignancies: An evidence-based update. Ophthalmology 108: 2088-2098, 2099-2100, 2001.

2. Wang L, Shan Y, Dai X, You N, Shao J, Pan X, Gao T and Ye J: Clinicopathological analysis of 5146 eyelid tumours and tumour-like lesions in an eye centre in South China, 2000-2018: A retrospective cohort study. BMJ Open 11: e041854, 2021.

3. Kaliki S, Ayyar A, Dave TV, Ali MJ, Mishra DK and Naik MN: Sebaceous gland carcinoma of the eyelid: Clinicopathological features and outcome in Asian Indians. Eye (Lond) 29: 958-963 2015.

4. Watanabe A, Sun MT, Pirbhai A, Ueda K, Katori N and Selva D: Sebaceous carcinoma in Japanese patients: Clinical presentation, staging and outcomes. Br J Ophthalmol 97: 1459-1463, 2013.

5. Cicinelli MV and Kaliki S: Ocular sebaceous gland carcinoma: An update of the literature. Int Ophthalmol 39: 1187-1197, 2019.

6. Esmaeli B, Nasser QJ, Cruz H, Fellman M, Warneke CL and Ivan D: American Joint Committee on Cancer $\mathrm{T}$ category for eyelid sebaceous carcinoma correlates with nodal metastasis and survival. Ophthalmology 119: 1078-1082, 2012.

7. Zhou C, Shi Y, Chai P, Wu F, Xia W, He X, Shi Y, Huang H, Jia R and Fan X: Contemporary update of overall prognosis and nomogram to predict individualized survival for Chinese patients with eyelid sebaceous carcinoma. EBioMedicine 36: 221-228, 2018

8. Shields JA, Demirci H, Marr BP, Eagle RC Jr and Shields CL: Sebaceous carcinoma of the eyelids: Personal experience with 60 cases. Ophthalmology 111: 2151-2157, 2004.

9. Hirano T, Yunoki T, Furusawa Y, Tabuchi Y and Hayashi A: Bioinformatics analysis of the microRNA-mRNA network in sebaceous gland carcinoma of the eyelid. Mol Med Rep 23: 23, 2021.

10. Bhardwaj M, Sen S, Chosdol K, Sharma A, Pushker N, Kashyap S, Bakhshi S and Bajaj MS: miRNA-200c and miRNA-141 as potential prognostic biomarkers and regulators of epithelial-mesenchymal transition in eyelid sebaceous gland carcinoma. Br J Ophthalmol 101: 536-542, 2017.

11. Dang X, Lin H, Li Y, Guo X, Yuan Y, Zhang R, Li X, Chai D and Zuo Y: MicroRNA profiling in BEAS-2B cells exposed to alpha radiation reveals potential biomarkers for malignant cellular transformation. Toxicol Res (Camb) 9: 834-844, 2020.

12. Kocyigit I, Taheri S, Sener EF, Eroglu E, Ozturk F, Unal A, Korkmaz K, Zararsiz G, Sipahioglu MH, Ozkul Y, et al: Serum micro-rna profiles in patients with autosomal dominant polycystic kidney disease according to hypertension and renal function. BMC Nephrol 18: 179, 2017.

13. Adams JC and Lawler J: The thrombospondins. Cold Spring Harb Perspect Biol 3: a009712, 2011.

14. Huang C, Zhou X, Li Z, Liu H, He Y, Ye G and Huang K: Downregulation of thrombospondin-1 by DNA hypermethylation is associated with tumor progression in laryngeal squamous cell carcinoma. Mol Med Rep 14: 2489-2496, 2016.

15. Huang WT, Chong IW, Chen HL, Li CY, Hsieh CC, Kuo HF, Chang CY, Chen YH, Liu YP, Lu CY, et al: Pigment epithelium-derived factor inhibits lung cancer migration and invasion by upregulating exosomal thrombospondin 1. Cancer Lett 442: 287-298, 2019.

16. Lin T,Zhu L, Zhou B, Xie L, Lv J, Dong L and He Y: Establishment and characterization of a cell line from human adenoid cystic carcinoma of the lacrimal glands and a nude mouse transplantable model. Oncol Rep 33: 2797-2806, 2015.

17. Livak KJ and Schmittgen TD: Analysis of relative gene expression data using real-time quantitative PCR and the 2(-Delta Delta C(T)) method. Methods 25: 402-408, 2001.

18. Agarwal V, Bell GW, Nam JW and Bartel DP: Predicting effective microRNA target sites in mammalian mRNAs. eLife 4: 4,2015
19. Liu W and Wang X: Prediction of functional microRNA targets by integrative modeling of microRNA binding and target expression data. Genome Biol 20: 18, 2019.

20. Pe'er J: Pathology of eyelid tumors. Indian J Ophthalmol 64: 177-190, 2016.

21. Satomura H, Ogata D, Arai E and Tsuchida T: Dermoscopic features of ocular and extraocular sebaceous carcinomas. J Dermatol 44 1313-1316, 2017.

22. Muqit MM, Foot B, Walters SJ, Mudhar HS, Roberts F and Rennie IG: Observational prospective cohort study of patients with newly-diagnosed ocular sebaceous carcinoma. $\mathrm{Br} \mathrm{J}$ Ophthalmol 97: 47-51, 2013

23. Buitrago W and Joseph AK: Sebaceous carcinoma: The great masquerader: Emgerging concepts in diagnosis and treatment. Dermatol Ther 21: 459-466, 2008

24. Goto H, Tsubota K, Nemoto R, Ueda S, Umazume K, Usui Y and Matsumura $\mathrm{H}$ : Clinical features and prognosis of sebaceous carcinoma arising in the eyelid or conjunctiva. Jpn J Ophthalmol 64: 549-554, 2020

25. Owen JL, Kibbi N, Worley B, Kelm RC, Wang JV, Barker CA Behshad R, Bichakjian CK, Bolotin D, Bordeaux JS, et al: Sebaceous carcinoma: Evidence-based clinical practice guidelines. Lancet Oncol 20: e699-e714, 2019.

26. Tang S, Li S, Liu T, He Y, Hu H, Zhu Y, Tang S and Zhou H: MicroRNAs: Emerging oncogenic and tumor-suppressive regulators, biomarkers and therapeutic targets in lung cancer. Cancer Lett 502: 71-83, 2021.

27. Bladen JC, Wang J, Sangaralingam A, Moosajee M, Fitchett C, Chelala C, Beaconsfield M, O'Toole EA, Philpott MP and Ezra DG: MicroRNA and transcriptome analysis in periocular Sebaceous Gland Carcinoma. Sci Rep 8: 7531, 2018.

28. Tetzlaff MT, Curry JL, Yin V, Pattanaprichakul P, Manonukul J, Uiprasertkul M, Manyam GC, Wani KM, Aldape K, Zhang L, et al: Distinct pathways in the pathogenesis of sebaceous carcinomas implicated by differentially expressed microRNAs. JAMA Ophthalmol 133: 1109-1116, 2015.

29. Tetzlaff MT, Singh RR, Seviour EG, Curry JL, Hudgens CW, Bell D, Wimmer DA, Ning J, Czerniak BA, Zhang L, et al: Next-generation sequencing identifies high frequency of mutations in potentially clinically actionable genes in sebaceous carcinoma. J Pathol 240: 84-95, 2016.

30. Bao Y, Selfridge JE, Wang J, Zhao Y, Cui J, Guda K, Wang Z and Zhu Y: Mutations in TP53, ZNF750, and RB1 typify ocular sebaceous carcinoma. J Genet Genomics 46: 315-318, 2019.

31. Tetzlaff MT, Curry JL, Ning J, Sagiv O, Kandl TL, Peng B, Bell D, Routbort M, Hudgens CW, Ivan D, et al: distinct biological types of ocular adnexal sebaceous carcinoma: HPV-driven and virus-negative tumors arise through nonoverlapping molecular-genetic alterations. Clin Cancer Res 25: 1280-1290, 2019.

32. Zaslavsky A, Baek KH, Lynch RC, Short S, Grillo J, Folkman J, Italiano JE Jr and Ryeom S: Platelet-derived thrombospondin-1 is a critical negative regulator and potential biomarker of angiogenesis. Blood 115: 4605-4613, 2010.

33. Yang W, Li W, Pan F, Yang XY, Zhao X, Qin L and Pan Y: TSP-1 as a novel biological marker of tumor vasculature normalization in colon carcinoma induced by Endostar. Oncol Lett 19: 2107-2114, 2020

34. Sevilla-Montero J, Bienes-Martínez R, Labrousse-Arias D, Fuertes-Yebra E, Ordóñez Á and Calzada MJ: pVHL-mediated regulation of the anti-angiogenic protein thrombospondin-1 decreases migration of clear cell renal carcinoma cell lines. Sci Rep 10: 1175, 2020.

35. Rouanne M, Adam J, Goubar A, Robin A, Ohana C, Louvet E, Cormier J, Mercier O, Dorfmüller P, Fattal S, et al: Osteopontin and thrombospondin-1 play opposite roles in promoting tumor aggressiveness of primary resected non-small cell lung cancer. BMC Cancer 16: 483, 2016.

36. Pal SK, Nguyen CT, Morita KI, Miki Y, Kayamori K, Yamaguchi A and Sakamoto K: THBS1 is induced by TGFB1 in the cancer stroma and promotes invasion of oral squamous cell carcinoma. J Oral Pathol Med 45: 730-739, 2016.

37. Cen J, Feng L, Ke H, Bao L, Li LZ, Tanaka Y, Weng J and Su L Exosomal thrombospondin-1 disrupts the integrity of endothelial intercellular junctions to facilitate breast cancer cell metastasis Cancers (Basel) 11: 11, 2019.

This work is licensed under a Creative Commons Attribution-NonCommercial-NoDerivatives 4.0 International (CC BY-NC-ND 4.0) License. 